\title{
Data Preparation Requirements for Modeling Wind Turbines with ADAMS ${ }^{\circledR}$
}

Marshall L. Buhl, Jr.

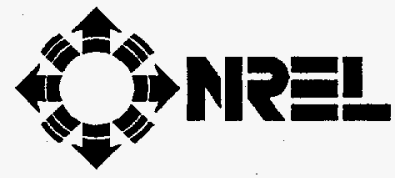

National Renewable Energy Laboratory 1617 Cole Boulevard Golden, Colorado 80401-3393

A national laboratory of the U.S. Department of Energy Managed by Midwest Research Institute for the U.S. Department of Energy under contract No. DE-AC36-83CH10093

Work performed under task number WE429150

December 1994 


\section{NOTICE}

This report was prepared as an account of work sponsored by an agency of the United States government. Neither the United States government nor any agency thereof, nor any of their employees, makes any warranty, express or implied, or assumes any legal liability or responsibility for the accuracy, completeness, or usefulness of any information, apparatus, product, or process disclosed, or represents that its use would not infringe privately owned rights. Reference herein to any specific commercialproduct, process, or service by trade name, trademark, manufacturer, or otherwise does not necessarily constitute or imply its endorsement, recommendation, or favoring by the United States government or any agency thereof. The views and opinions of authord expressed herein do not necessarily state or reflect those of the United States government or any agency thereof.

Available to DOE and DOE contractors from:

Office of Scientific and Technical Information (OSTI)

P.O. Box 62

Oak Ridge, TN 37831

Prices available by calling (615) 576-8401

Available to the public from:

National Technical information Service (NTIS)

U.S. Department of Commerce

5285 Port Royal Road

Springfield, VA 22161

(703) $487-4650$ 


\section{DISCLAIMER}

\section{Portions of this document may be illegible in electronic image products. Images are produced from the best available original document.}




\section{MODELING WIND TURBINES WITH ADAMS ${ }^{\circledR}$}

\section{INTRODUCTION}

What follows are guidelines for the kind of information that you will need to model a "complete" wind turbine with $A D A M S$. The information here is not at all exhaustive. It does, however, represent the collective knowledge of two years of experience gained by National Renewable Energy Laboratory (NREL) engineers while using $A D A M S$ to model wind turbines. If you save the following information as you design a new turbine, you will have an excellent start on an $A D A M S$ model. Please remember that the more accurate your input data, the better your results will be. You will have to make the tradeoff between the required effort to improve your input data and the benefits of a more accurate simulation.

We break the turbine into each of its major subsystems and describe them in detail below. We attach a table of parameters to the end of the document to make it easier for you to keep track of your data requirements.

\section{TOWER:}

You can model your tower as a rigid structure that has no effect on the system dynamics or as a collection of lumped masses separated by flexible, force-at-a-distance "beams" or "fields."

For the first method, you model the tower as part of the ground; the geometry of the tower is the only information needed. We often do this to simplify the model for special cases, or to check other subsystems.

NREL engineers usually use the second method to model towers. The difference between beams and fields is that beams assume uniform properties between the two lumped masses. Most towers have only a slight taper (if any), so we believe that beams are sufficiently accurate and that fields do not merit the additional modeling efforts they require. If your tower is highly tapered, you should consider using fields.

Whether you use beams or fields to model your tower, break the tower into five to ten lumped masses, depending upon stiffness (you will not need as many for stiffer towers). For each mass, you need the location of the centers of mass, masses, and mass moments of inertia about all three axes.

For beams, $A D A M S$ requires the cross sectional areas, the area moments of inertia about each axis, Young's modulus, and the shear modulus for each beam. ADAMS uses these values to compute a stiffness matrix.

When using fields, you must specify a stiffness matrix instead of the inertias, areas, and moduli-though you will probably need them to create your own stiffness matrix.

For both beams and fields, you need to supply beam lengths and a damping "ratio." $A D A M S$ multiplies each element of the stiffness matrix by the damping ratio to generate a damping matrix. You can supply your own damping matrix if you desire.

If you have a guyed tower, you will need to know the geometry, spring constant, damping constant, and the pretension in the guys to model the guys as massless springs. If the inertia of the guys is large enough to affect overall system dynamics, you may want to model them as a flexible subsystem. If so, follow the lumpedmass approach outlined above, but use spring/dampers between the masses instead of beams or fields.

Even though it has no impact upon dynamics, you will probably want to generate a graphic that approximates the shape of the tower for animation purposes. For this, you will need the tower geometry. The current version of $A D A M S$ can draw lines, spheres, cylinders, frustums, boxes, and other simple graphics objects.

\section{NACELlE:}

We have modeled nacelles as rigid bodies. We do not include in the nacelle the parts of the drive train we wish to model separately. You will need to know the mass, center of mass, mass moments of inertia, and geometry of the nacelle. Even if you lock or drive the machine yaw, this information will affect tower modes. You will also need an accurate measurement of the location of the yaw bearing and the low speed shaft (LSS).

You can obtain the mass and mass moments of inertia by measuring an assembled nacelle without parts (such as the LSS) that you will model separately. It is possible, but not easy, to measure the center of mass and inertias.

Another method is to compute the total nacelle properties by adding in the effects of each component. If appropriate, treat each component as a single-point mass. This is probably the easiest method if you have all the component masses and locations. You may want to compare the total computed mass to a weighed nacelle and modify the numbers to get them to agree.

You can specify the nacelle shroud geometry to permit animated graphics.

\section{DRIVE TRAIN:}

If you want to model your turbine with steady-state rotor motion, you can connect the hub to the nacelle with 
a revolute joint (hinge) and drive it with a MOTION statement. You will need only the rotor revolutions per minute (RPM), the shaft tilt, and the location of the attachment of the hub to the nacelle.

We approximate the generator by using Thevenin's Theorem (Fitzgerald, Kingsley, and Umans 1983, 424427) to relate shaft slip to torque. This theorem really only applies for operation near steady state, but you can use it as a crude approximation for startups. For this, you will need to get some values off the nameplate and others from the manufacturer. From the nameplate, get the actual RPM, line-to-line voltage, and frequency. From the manufacturer, get the stator resistance, stator leakage reactance, rotor resistance, rotor leakage reactance, magnetizing reactance, and iron-core-loss resistance.

For the gearbox, ADAMS does have a standard gear, but it is a perfect one; it does not model backlash. You will need to know the gear reduction to model this. Using user-written subroutines, you can create models of your generator and/or gear box in even greater detail.

You can model shafts as rigid parts; as simple, rotational spring/dampers assuming the shaft does not bend; or as distributed-mass, flexible beams. For a rigid shaft you need only mass, center of mass, mass moments of inertia, shaft tilt, and the point of attachment. For a spring/damper shaft, you will also need the spring and damping constants of the shaft. To model a fully flexible shaft, you will have to divide it into lumped masses in a fashion similar to the flexible tower.

HUB:

For a three-bladed rotor, you can adequately model the hub as a rigid body. For this, you need the mass, center of mass, and mass moments of inertia. You will also need the attachment points for the LSS and the blades. You will need the rotor precone angle to create the blade attachment joints. As always, geometry information is useful for animations.

For a two-bladed, teetering rotor, in addition to the data required for three-bladed rotors, you will need the undersling, the delta- 3 angle, the contact angle for the teeter mechanism, and the stiffness and damping of the teeter stops. You may want to use a flexible model of the hub. For this, use the tower-modeling approach.

\section{BLADES:}

Blades are the most difficult part of the turbine to model. You will need to discretize the blades into lumped masses and specify their locations. You can connect the masses with rigid joints, flexible beams, or flexible fields. At NREL, we model our blades as both rigid and as flexible beams. Having the option to make the blades rigid is useful for checking out aerodynamics.

To make a rigid blade model, divide the blade into discrete masses and specify the centers of mass, mass moments of inertia, twist, chord, and thickness. You must also specify the pitch of the blade.

To create a flexible-beam blade model, augment the above information. Using the "lumped-mass" approach, divide the blade into beams that connect the discrete masses. As for the tower, $A D A M S$ needs to know the beam lengths, their cross-sectional areas, area moments of inertia, Young's moduli, shear moduli, and the damping ratios or matrices. If the elastic axis does not pass through the centers of mass, you should specify this offset. To use fields, you must generate the stiffness matrix instead of area moments of inertia and moduli.

A modal survey of your blade (if available) will considerably improve the accuracy of the model. You can use $A D A M S /$ Linear to generate eigenvalues to compare to the modal survey. By tweaking the moduli, area moments of inertia, and damping matrices, you can adjust your blade model's natural frequencies and damping to reflect the modal survey. If you decide to use fields you will have the stiffness and damping matrices to work with.

Some turbines have tip brakes. If yours does, you will need their masses and locations. You may also want to specify their geometry for graphics output.

\section{AERODYNAMICS:}

We use aerodynamics subroutines developed at the University of Utah to compute normal and tangential forces applied to points along the blade. They wrote a user's guide (Hansen and Davis 1994) that lists input data requirements for the aerodynamics routines. Additionally, if the centers of pressure are not aligned with the centers of mass you will need their offsets when placing the force vectors in $A D A M S$.

\section{WINDS:}

The University of Utah aerodynamics routines uses wind in three ways. First, you can specify constant hubheight, horizontal and vertical wind speeds, and horizontal and vertical wind shears. It is also possible to specify a file that contains time-varying winds, and you can change the code to read the kind of information that interests you. The third method is to specify files that contain time-varying $u, v$, and $w$ component wind speeds for a grid that encompasses the rotor disk. This last method uses the output of NREL's SNLWIND-3D turbulence model.

\section{REFERENCES:}

Hansen, Craig; Davis, Dean. 1994. User's Guide to AeroDyn the Wind Turbine Aerodynamics Model for $A D A M S \otimes$. Version 9.0. Salt Lake City: University of Utah.

Fitzgerald, A. E.; Kingsley, Charles Jr.; Umans, S. D. (1983). Electric Machinery. 4th ed. New York: McGraw-Hill Book Company. 


\section{TABLE OF PARAMETERS \\ FOR \\ MODELING WIND TURBINES WITH ADAMS}

TOWER:

Rigid

Tower height (location of yaw bearing)

Tower geometry (for graphics)

Lumped-Mass Flexible Beams

Tower height (location of yaw bearing)

Mass of lumped masses

Locations of lumped masses

Mass moments of inertia of lumped masses

Beam lengths

Beam cross-sectional areas

Beam area moments of inertia

Young's moduli

Shear moduli

Damping "ratio" or damping matrix

Tower geometry (for graphics)

Lumped-Mass Flexible Fields

Tower height (location of yaw bearing)

Mass of lumped masses

Locations of lumped masses

Mass moments of inertia of lumped masses

Beam lengths

Stiffness matrix

Damping "ratio" or damping matrix

Tower geometry (for graphics)

Massless Spring-Damper Guys

Guy geometry (attachment points and length)

Spring constant

Damping constant

Guy pretension

\section{Lumped-Mass Flexible Guys}

Guy geometry (attachment points)

Mass of lumped masses

Locations of lumped masses

Mass moments of inertia of lumped masses

Spring constants for each segment

Damping constants for each segment

Guy pretensions for each segment
NACELLE:

If you choose to measure the total nacelle

Total nacelle mass

Mass moments and products of inertia

Nacelle center of mass

Location of yaw bearing

Location of beginning of separately modeled drive train

Nacelle shroud geometry (for graphics)

If you choose to derive properties from components

Component masses

Mass moments and products of inertia of large parts

Component centers of mass

Nacelle shroud geometry (for graphics)

DRIVE TRAIN:

Steady-state rotor motion

Rotor RPM

Shaft tilt

Hub attachment location

Steady-state generator

Actual RPM

Line-to-line voltage

Frequency

Stator resistance

Stator leakage reactance

Rotor resistance

Rotor leakage reactance

Magnetizing reactance

Iron core loss resistance

Perfect Gearbox

Gear ratio

Rigid Shafts

Shaft mass

Center of mass

Mass moments of inertia

Shaft tilt

Attachment points

Shaft diameter and length (for graphics) 


\author{
Spring/damper shafts \\ Shaft mass \\ Center of mass \\ Mass moments of inertia \\ Shaft tilt \\ Attachment points \\ Spring constant \\ Damping Constant \\ Shaft diameter and length (for graphics) \\ Flexible shafts \\ Mass of lumped masses \\ Locations of lumped masses \\ Mass moments of inertia of lumped masses \\ Beam lengths \\ Beam cross-sectional areas \\ Beam area moments of inertia \\ Young's moduli \\ Shear moduli \\ Damping "ratio" or damping matrix \\ Shaft tilt \\ Attachment points \\ Shaft diameter and lengths (for graphics)
}

HuB:

Rigid hubs

Mass

Center of mass

Mass moments of inertia

Location of LSS attachment

Location of Blade attachments

Geometry information (for graphics)

Flexible hubs

Hub geometry for animations

Mass of lumped masses

Locations of lumped masses

Mass moments of inertia of lumped masses

Beam lengths

Beam cross-sectional areas

Beam area moments of inertia

Young's moduli

Shear moduli

Damping "ratio" or damping matrix

Geometry information (for graphics)

\section{BLADES:}

\section{Rigid blades}

Mass of lumped masses

Centers of mass

Mass moments of inertia

Blade structural twist

Blade aerodynamic twist

Blade chord and thickness (for graphics)

Tip brake mass

Tip brake center of mass

Tip brake moments of inertia
Flexible beam blades

Mass of lumped masses

Centers of mass

Mass moments of inertia

Blade structural twist

Blade aerodynamic twist

Beam lengths

Beam cross-sectional areas

Beam area moments of inertia

Young's modulus for each beam

Shear modulus for each beam

Damping ratios or matrices

Eigenvectors from modal survey if available

Blade chord and thickness (for graphics)

Tip brake mass

Tip brake center of mass

Tip brake moments of inertia

Flexible field blades

Mass of lumped masses

Centers of mass

Mass moments of inertia

Blade structural twist

Blade aerodynamic twist

Beam lengths

Stiffness matrix for each beam

Damping ratios or matrices

Eigenvectors from modal survey if available

Blade chord and thickness (for graphics)

Tip brake mass

Tip brake center of mass

Tip brake moments of inertia

\section{AERODYNAMICS:}

University of Utah Aerodynamics

See University of Utah user's guides

Centers of pressures

WinDS:

Steady wind

Horizontal wind speed

Vertical wind speed

Horizontal wind shear ratio (linear)

Vertical wind shear (ratio for linear, exponent for power-law)

Time varying wind

Horizontal wind speed time history

Vertical wind speed time history

Wind direction time history

Horizontal wind shear ratio (linear) time history

Vertical wind shear (ratio for linear, exponent for power-law) time history

Full-field turbulent wind

Time history of $u, v$, and $w$ translational component winds (generated by SNLWIND-3D) 